\title{
Association between diabetes mellitus with metabolic syndrome and diabetic microangiopathy
}

\author{
XIAOYAN ZHANG, XIAOLI CUI, FENGHUA LI, SHUO WANG, XINYU LIU, \\ LICHAO HUI, NA SONG and NANNAN LI
}

\begin{abstract}
Department of Endocrinology, First Affiliated Hospital, Liaoning Medical College, Jinzhou, Liaoning 121001, P.R. China
\end{abstract}
Received January 12, 2014; Accepted June 13, 2014

DOI: $10.3892 /$ etm.2014.1992

\begin{abstract}
The aim of this study was to investigate the association between diabetes mellitus (DM), mainly type II, with metabolic syndrome (MS) and diabetic nephropathy (DN)/diabetic retinopathy (DR). Based on the analysis of the prevalence of MS, patients with DM were divided into MS and non-MS groups according to the presence or absence of MS. The correlation between DN, DR and certain factors, including gender, age, disease duration and the presence or absence of a family history of MS, were analyzed. The prevalence of MS among the patients with DM was $62.50 \%$. The prevalence of DN was $55.33 \%$ in the MS group and that of DR was $26.00 \%$. DN was positively correlated with age, gender, blood pressure, triglyceride (TG), low-density lipoprotein cholesterol (LDL-C) and blood uric acid. DR was positively correlated with traceable disease duration and LDL-C. In conclusion, DM occurred more frequently in concurrence with MS than without MS, and the prevalence of DN/DR in the MS group was higher than that in the non-MS group. Age, gender, blood pressure, TG, LDL-C and blood uric acid were risk factors for DN and the traceable disease duration and LDL-C were risk factors for DR.
\end{abstract}

\section{Introduction}

At present, diabetes mellitus (DM) is a serious issue in China, and complications associated with the disease are a serious threat to human health. Diabetic nephropathy (DN) and diabetic retinopathy (DR) are the main microvascular complications, causing kidney failure and blindness, respectively. Metabolic syndrome (MS) is a group of metabolic disorders centered around insulin resistance (IR), with basic features including abnormal glucose metabolism, central obesity, lipid disorders and hypertension. The characteristics of MS vary

Correspondence to: Dr Xiaoyan Zhang, Department of Endocrinology, First Affiliated Hospital, Liaoning Medical College, 2 Wuduan Renmin Street, Jinzhou, Liaoning 121001, P.R. China E-mail: xyxlcn@163.com

Key words: diabetes, diabetic microangiopathy, metabolic syndrome among different ethnic groups on a global scale and among different provinces within the same country. However, the overall prevalence of MS has increased.

The disease causes damage to the vital organs of the body. Although numerous factors lead to atherosclerosis and clinical cardiovascular events, the occurrence of MS is of particular importance; this has been confirmed in a number of studies described below (1). The National Cholesterol Education Program Adult Treatment Panel Third Guide (NCEP-ATP III) reported that the main clinical outcomes of MS are cardiovascular disease (CVD) and the aggravation of the occurrence and development of DN, DR and other microvascular diseases. Gluckman et al (2) believe that insulin resistance in the metabolic reaction develops in adults due to a poor prenatal development environment (such as malnutrition). The study thus speculated that growth in the adaptation to a bad developmental environment in the period of developmental plasticity can be generated by a permanent poor development environment, and that these genes could be susceptibility genes to diabetes or other metabolic diseases, thereby increasing the risk for diabetes in adults. Additionally, the prevalence of MS is expected to increase in China due to its high ageing population, and it is believed that it may bring heavy economic burden to families and the society, therefore, the prevention and treatment of MS is something that is required to be investigated (3). However, few conclusive studies exist on the association between DM with MS and diabetic microangiopathy. Thus, the associations between DM with MS and DN/DR were further explored in the present study by analyzing the hospital data from 240 patients recruited for the study in Jinzhou, China.

\section{Patients and methods}

Study subjects. Data from 240 patients with diabetes, hospitalized in the Department of Endocrinology of the First Affiliated Hospital of Liaoning Medical College (Jinzhou, China), were collected between March and November, 2012. The study population comprised 135 males and 105 females, aged 14-91 years, with a mean age of 55.6 \pm 11.1 years. This study was conducted in accordance with the Declaration of Helsinki, and with approval from the Ethics Committee of Liaoning Medical College. Written informed consent was obtained from all participants. 
Inclusion criteria

$D M$. The inclusion criteria for $\mathrm{DM}$ were designed in accordance with the 1999 World Health Organization (WHO) diagnostic criteria for diabetes (4). Patients who exhibited the following characteristics were included: i) Typical symptoms of diabetes (polyuria, polydipsia and unexplained weight loss); ii) random blood glucose levels $\geq 11.1 \mathrm{mmol} / \mathrm{l}$; iii) fasting plasma glucose levels $\geq 7.0 \mathrm{mmol} / 1$ or $2 \mathrm{~h}$ post-challenge glucose levels (hPG) in an oral glucose tolerance test $\geq 11.1 \mathrm{mmol} / 1$. Any atypical symptoms were confirmed on a different day.

$M S$. The inclusion criteria for MS were designed in accordance with the diagnostic criteria for MS proposed by the Chinese Medical Association Diabetes Society (CDS) in 2004 (5): i) Being overweight and/or obese (body mass index $\geq 25 \mathrm{~kg} / \mathrm{m}^{2}$ ); ii) hyperglycemia [fasting plasma glucose levels $\geq 6.1 \mathrm{mmol} / 1(110 \mathrm{mg} / \mathrm{dl})$ and $/$ or $2 \mathrm{hPG} \geq 7.8 \mathrm{mmol} / 1$ $(140 \mathrm{mg} / \mathrm{dl})$ and/or patients have been diagnosed as diabetic and treated]; iii) hypertension [blood pressure (BP) $\geq 140 / 90 \mathrm{mmHg}$ and/or patients have been diagnosed as hypertensive and treated]; iv) dyslipidemia [fasting triglyceride (TG) levels $\geq 1.7 \mathrm{mmol} / \mathrm{l}(150 \mathrm{mg} / \mathrm{dl})$ and/or fasting high-density lipoprotein cholesterol (HDL-C) levels $<0.9 \mathrm{mmol} / 1$ ( $35 \mathrm{mg} / \mathrm{dl}$ ) (male) or $<1.0 \mathrm{mmol} / 1$ (39 mg/dl) (female)]. Three or all of the four standards being fulfilled led to a diagnosis of MS.

$D N$. According to the diagnostic standards of DN in the Mogensen DN diagnostic criteria (6), DN was divided into five phases: Phase I, a high glomerular filtration period characterized by renal hypertrophy, increasing glomerular filtration rate (GFR) and normal urinary albumin excretion rate (UAER); Phase II, a normal albuminuria period with no obvious clinical manifestations, normal UAER $(<20 \mu \mathrm{g} / \mathrm{min})$ in a resting state and slightly increased UAER in the stress state, and normal or slightly elevated GFR; Phase III, persistent microalbuminuria (MAU) with clinical features such as UAER of $20-200 \mu \mathrm{g} / \mathrm{min}$, urinary albumin quantification of $30-300 \mathrm{mg}$ in $24 \mathrm{~h}$, negative for urine protein in a routine urine test and normal GFR; Phase IV, a clinical proteinuria period with persistent positive results for urine protein, $24 \mathrm{~h}$ urine protein $>0.5 \mathrm{~g}$, UAER $>200 \mu \mathrm{g} / \mathrm{min}$ and a gradual decrease in GFR; Phase V, end-stage, renal failure with further decline in GFR, GFR $<15 \mathrm{ml} / \mathrm{min}$ or dialysis, often with heavy proteinuria.

$D R$. The DR diagnostic criteria used were based on the diagnostic criteria developed by the Third Sector National Eye Conference in 1985; the staging of DR is shown in Table I.

Grouping. The subjects were initially divided into two groups according to gender (135 males and 105 females), and the prevalence of MS, DN and DR in the two groups was calculated. Patients were then divided into the MS and non-MS groups based on whether they suffered from MS or not. The prevalence of DR and DN in the two groups was calculated, as well as the prevalence of DN and DR in the two different gender groups.

Detection index. Information such as age, gender and traceable duration of diabetes of all the subjects was collected following admission. The BP, height and weight of the subjects were measured by the uniformly trained personnel. Fasting venous plasma glucose was measured by the hexokinase method and the enzyme method was utilized to measure fasting TG and total

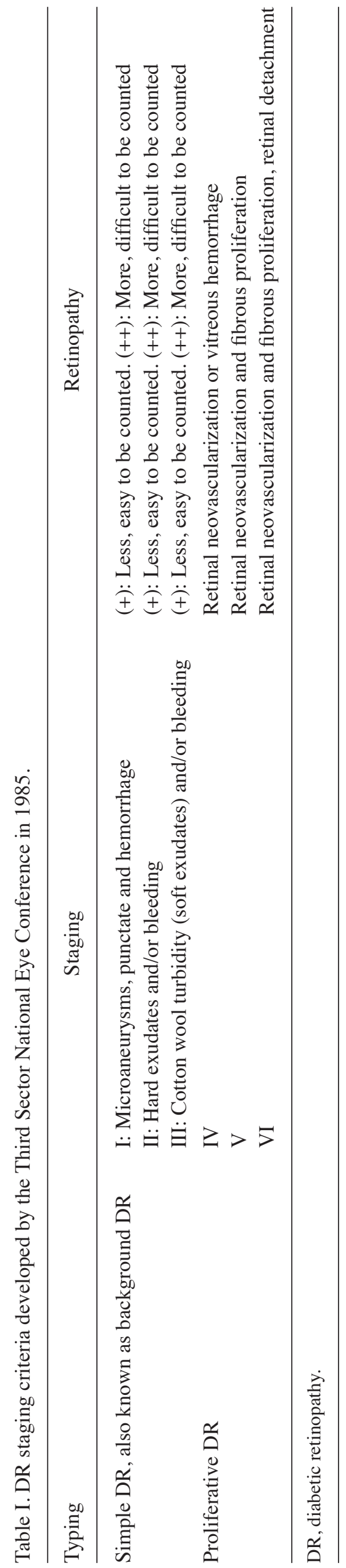


Table II. Univariate logistic regression analysis between diabetic nephropathy and single factors.

\begin{tabular}{|c|c|c|c|c|c|c|}
\hline Variable & $\beta$ & SE & Wald $\left(\chi^{2}\right)$ & P-value & OR & $95 \%$ CI OR \\
\hline Age & 0.023 & 0.009 & 6.152 & 0.013 & 1.023 & $1.005-1.041$ \\
\hline Gender & 0.033 & 0.062 & 4.211 & 0.041 & 0.849 & $0.388-1.085$ \\
\hline Traceable disease duration & 0.035 & 0.021 & 2.664 & 0.130 & 1.035 & 0.993-1.079 \\
\hline Family history & 0.064 & 0.186 & 0.852 & 0.356 & 0.768 & $0.438-1.345$ \\
\hline SBP & 0.037 & 0.008 & 22.245 & $<0.001$ & 1.038 & $1.022-1.054$ \\
\hline DBP & 0.041 & 0.013 & 10.158 & 0.001 & 1.042 & $1.016-1.068$ \\
\hline BMI & 0.019 & 0.035 & 0.280 & 0.597 & 1.019 & $0.951-1.091$ \\
\hline TG & 0.188 & 0.081 & 5.456 & 0.019 & 1.207 & $1.031-1.413$ \\
\hline $\mathrm{TC}$ & 0.005 & 0.008 & 0.395 & 0.530 & 0.995 & $0.978-1.011$ \\
\hline LDL-C & 0.360 & 0.157 & 5.276 & 0.022 & 1.433 & $1.054-1.949$ \\
\hline HDL-C & -0.018 & 0.028 & 0.404 & 0.025 & 0.983 & $0.931-1.037$ \\
\hline HbA1c & 0.023 & 0.035 & 0.435 & 0.510 & 0.977 & $0.911-1.047$ \\
\hline Blood uric acid & 0.003 & 0.001 & 5.178 & 0.023 & 1.003 & $1.000-1.006$ \\
\hline $\mathrm{C}$ peptide & 0.040 & 0.063 & 0.401 & 0.526 & 1.041 & 0.919-1.179 \\
\hline Blood sugar & 0.010 & 0.012 & 0.650 & 0.420 & 1.010 & $0.986-1.034$ \\
\hline
\end{tabular}

SE, standard error; OR, odds ratio; CI, confidence interval; SBP, systolic blood pressure; DBP, diastolic blood pressure; BMI, body mass index; TG, triglyceride; TC, total cholesterol; LDL-C, low-density lipoprotein cholesterol; HDL-C, high-density lipoprotein cholesterol; HbA1c, glycosylated hemoglobin A1c; C peptide, connecting peptide.

cholesterol (TC). Fasting HDL-C and low-density lipoprotein cholesterol (LDL-C) were measured using the enzyme-modified method and measurements of glycosylated hemoglobin A1c (HbAlc) were obtained using high-performance liquid chromatography. Enzymatic kinetics, electrochemiluminescence and immunonephelometry were used to obtain measurements of serum uric acid, serum connecting peptide and urinary albumin, respectively, and $24 \mathrm{~h}$ urinary protein was measured using chromometry. Ophthalmoscopy was performed on selected patients by the same ophthalmologist in the First Affiliated Hospital of Liaoning Medical College, and fundus angiography was performed when necessary.

Statistical analysis. All data were processed using the SPSS 17.0 statistical software package (SPSS, Inc., Chicago, IL, USA). Age is expressed as the mean \pm standard deviation. Comparisons among the prevalence of MS, DN and DR were performed by the $\chi^{2}$ test with independent samples. The associations between MS and DN/DR are expressed by non-conditional logistic regression analysis. $\mathrm{P}<0.05$ was considered to be statistically significant.

\section{Results}

Prevalence of MS in different gender groups. The prevalence of MS among the diabetic patients was $62.50 \%$ (150 cases), and the prevalence of MS among the males and females was $66.67 \%$ (90 cases) and 57.14\% (60 cases), respectively. The difference in prevalence between the two gender groups was not statistically significant $\left(\chi^{2}=2.286, \mathrm{P}>0.10\right)$.

Prevalence of DN or DR in different gender groups. The prevalence for DN among the male and female patients were $53.33 \%$
( 72 cases) and $40.00 \%$ (42 cases), respectively; the difference in prevalence between the two groups was statistically significant $\left(\chi^{2}=4.211, \mathrm{P}<0.05\right)$. The prevalence for $\mathrm{DR}$ among the male and female patients were $22.22 \%$ (30 cases) and $23.81 \%$ ( 25 cases); the difference in prevalence between the two groups was not statistically significant $\left(\chi^{2}=0.084, \mathrm{P}>0.05\right)$.

Prevalence of DN or DR in the MS and non-MS groups. The prevalence of DN in the MS group was $55.33 \%$ (83 cases), which was significantly higher than that in the non-MS group (34.44\%, 31 cases). The difference in prevalence between the two groups was statistically significant $\left(\chi^{2}=9.842, \mathrm{P}<0.005\right)$. The prevalence of DR was $26.00 \%$ (39 cases) in the MS group and $17.78 \%$ (16 cases) in the non-MS group; the difference in prevalence between the two groups was not statistically significant $\left(\chi^{2}=2.153, \mathrm{P}>0.05\right)$.

Prevalence of DN among the different genders in the MS and non-MS groups. The prevalence of DN among the males and females in the MS group was $58.89 \%$ (53 cases) and $50.00 \%$ (30 cases), respectively; the difference in prevalence between the two groups was not statistically significant $\left(\chi^{2}=1.151\right.$, $\mathrm{P}>0.05)$. The prevalence of DN among the males and females in the non-MS group was $42.22 \%$ (19 cases) and $26.67 \%$ (12 cases), respectively; this difference was not statistically significant $\left(\chi^{2}=2.411, \mathrm{P}>0.05\right)$.

Prevalence of DR among the different genders in the MS and non-MS groups. The prevalence of DR among the males and females in the MS group was $23.33 \%$ (21 cases) and $30.00 \%$ (18 cases), respectively; the difference in prevalence between the two groups was not statistically significant $\left(\chi^{2}=0.832\right.$, $\mathrm{P}>0.05)$. The prevalence of DR among the males and females 
Table III. Univariate logistic regression analysis between diabetic retinopathy and single factors.

\begin{tabular}{|c|c|c|c|c|c|c|}
\hline Variable & $\beta$ & SE & Wald $\left(\chi^{2}\right)$ & P-value & OR & $95 \%$ CI OR \\
\hline Age & 0.017 & 0.011 & 2.578 & 0.108 & 1.018 & $0.996-1.040$ \\
\hline Gender & 0.163 & 0.308 & 0.279 & 0.579 & 1.177 & $0.143-2.153$ \\
\hline Traceable disease duration & 0.128 & 0.026 & 24.223 & $<0.001$ & 1.136 & $1.080-1.196$ \\
\hline Family history & 0.028 & 0.172 & 2.843 & 0.092 & 0.534 & $0.257-1.107$ \\
\hline SBP & 0.010 & 0.008 & 1.625 & 0.202 & 1.010 & $0.995-1.025$ \\
\hline DBP & 0.006 & 0.014 & 0.194 & 0.660 & 1.006 & $0.979-1.034$ \\
\hline BMI & 0.030 & 0.042 & 0.514 & 0.474 & 1.030 & $0.950-1.118$ \\
\hline TG & 0.035 & 0.092 & 0.413 & 0.705 & 0.966 & $0.807-1.156$ \\
\hline $\mathrm{TC}$ & 0.203 & 0.113 & 3.225 & 0.073 & 1.225 & $0.982-1.528$ \\
\hline LDL-C & 0.386 & 0.181 & 4.562 & 0.033 & 1.471 & $1.032-2.097$ \\
\hline HDL-C & -0.021 & 0.058 & 0.127 & 0.721 & 0.979 & 0.874-1.098 \\
\hline HbA1c & 0.040 & 0.045 & 0.182 & 0.376 & 0.961 & 0.880-1.049 \\
\hline Blood uric acid & 0.001 & 0.101 & 0.611 & 0.434 & 1.001 & 0.998-1.004 \\
\hline $\mathrm{C}$ peptide & 0.023 & 0.073 & 0.095 & 0.757 & 1.023 & $0.886-1.181$ \\
\hline Blood sugar & 0.043 & 0.030 & 2.043 & 0.153 & 0.958 & $0.903-1.016$ \\
\hline
\end{tabular}

SE, standard error; OR, odds ratio; CI, confidence interval; SBP, systolic blood pressure; DBP, diastolic blood pressure; BMI, body mass index; TG, triglyceride; TC, total cholesterol; LDL-C, low-density lipoprotein cholesterol; HDL-C, high-density lipoprotein cholesterol; HbA1c, glycosylated hemoglobin A1c; C peptide, connecting peptide.

in the non-MS group was $20.00 \%$ ( 9 cases) and $5.56 \%$ ( 7 cases), respectively; this difference was not statistically significant $\left(\chi^{2}=0.304, \mathrm{P}>0.05\right)$.

Univariate analysis. Taking the presence or absence of DN or $\mathrm{DR}$ as the dependent variable and gender, age, traceable disease duration, systolic BP (SBP), diastolic BP (DBP), TG, HDL-C, $\mathrm{HbA} 1 \mathrm{c}$ or blood uric acid as independent variables, univariate logistic regression analysis was performed. DN was positively correlated with age, gender, SBP, DBP, TG, LDL-C and blood serum uric acid $(\mathrm{P}<0.05)$ and was negatively correlated with HDL-C $(\mathrm{P}<0.05)$ (Table II). DR was positively correlated with traceable duration and LDL-C $(\mathrm{P}<0.05$; Table III).

Multivariate analysis. Taking the presence or absence of DN or DR as the dependent variable and gender, age, traceable disease duration, SBP, DBP, TG, HDL-C, HbA1c or blood uric acid as independent variables, multivariate logistic regression analysis was performed. Age, uric acid and MS were risk factors for DN (Table IV), and traceable disease duration was a risk factor for DR (Table V).

\section{Discussion}

According to the available information, the prevalence of MS has increased significantly throughout the world. Furthermore, it is expected that the prevalence will increase exponentially in all countries in the next two centuries. MS is affected by numerous factors; as a result, the common features vary in different regions, as well as the used diagnostic criteria. The prevalence of MS in different regions also varies. In the Third National Health and Nutrition Examination Survey (NHANES III) in the USA, the NHANES III criteria were used as the diagnostic criteria for MS. The survey revealed that, in the USA, the prevalence of MS was $23.9 \%$ in adults $>20$ years old, with an approximately equal prevalence in the two genders (7). The prevalence of MS was significantly higher in adults $>50$ years old $(\sim 44 \%)$ and in obese subjects $(59.6 \%)$. In a study in the Middle East, International Diabetes Federation (IDF) and NCEP-ATPIII diagnostic criteria were used to study the prevalence of MS in diabetic and non-diabetic patients. According to the IDF and NCEP-ATPIII criteria, the prevalence of MS in non-diabetic patients was 29.0 and $31.5 \%$, respectively, while the prevalence of MS in diabetic patients was 63.4 and $64.2 \%$, respectively (8). NCEP-ATPIII diagnostic criteria were also used in a study in Canada, with the results showing that the total prevalence of MS was $19.1 \%$. The prevalence among female subjects was $20.5 \%$, which was higher than that among male subjects $(17.8 \%)$, but this difference was not significant (9). Jia et al (10) in China performed an MS survey with Chinese subjects aged 20-74 years in two communities in Shanghai. When the WHO criteria were used to define the standards, the prevalence of MS was $17.14 \%$, while the prevalence was $10.95 \%$ when the NCEP-ATPIII diagnostic criteria were used. A prevalence survey of MS among the elderly in five cities, including Chongqing, Jinan and Tianjin, was performed by Li et al (11), according to the diagnostic criteria for MS developed by the Joint Commission on Chinese Adult Dyslipidemia in 2007 and based on the recommendations of the CDS in 2004. The results showed that the crude prevalence of MS among the study population was $25.5 \%$, including rates of $23.2 \%$ for male and $26.9 \%$ for female subjects. In the present study, the hospitalization data from 240 patients with diabetes in Jinzhou were analyzed. The results showed that the prevalence of MS was $62.6 \%$, higher than that of the general population and similar to the prevalence of diabetes in 
Table IV. Multivariate logistic regression analysis between diabetic nephropathy and multiple factors.

\begin{tabular}{lcccccc}
\hline Variable & $\beta$ & SE & Wald $\left(\chi^{2}\right)$ & P-value & OR & 95\% CI OR \\
\hline Age & 0.028 & 0.011 & 6.856 & 0.009 & 1.029 & $1.007-1.051$ \\
Gender & 0.380 & 0.292 & 2.102 & 0.207 & 0.784 & $0.386-1.211$ \\
Traceable disease duration & 0.016 & 0.024 & 0.437 & 0.509 & 1.016 & $0.969-1.066$ \\
Blood uric acid & 0.003 & 0.001 & 4.463 & 0.035 & 1.003 & $1.000-1.006$ \\
MS & 0.847 & 0.294 & 8.282 & 0.004 & 2.333 & $1.310-4.156$
\end{tabular}

SE, standard error; OR, odds ratio; CI, confidence interval; MS, metabolic syndrome.

Table V. Multivariate logistic regression analysis between diabetic retinopathy and multiple factors.

\begin{tabular}{lcccccr}
\hline Variable & $\beta$ & SE & Wald $\left(\chi^{2}\right)$ & P-value & OR & $95 \%$ CI OR \\
\hline Age & 0.005 & 0.013 & 0.120 & 0.729 & 0.995 & $0.970-1.021$ \\
Gender & 0.144 & 0.347 & 0.172 & 0.679 & 1.155 & $0.585-2.281$ \\
Traceable disease duration & 0.134 & 0.029 & 21.479 & $<0.001$ & 1.143 & $1.080-1.210$ \\
Blood uric acid & 0.002 & 0.002 & 1.498 & 0.221 & 1.002 & $0.999-1.005$ \\
MS & 0.360 & 0.361 & 0.996 & 0.318 & 1.434 & $0.707-2.909$ \\
\hline
\end{tabular}

SE, standard error; OR, odds ratio; CI, confidence interval; MS, metabolic syndrome.

the Middle East. The prevalence was 66.67 and $57.14 \%$ for the male and female patients, respectively. With an increasingly aging society in China, the prevalence of MS is expected to further increase, which is likely to bring a heavy economic burden to families and society. Therefore, the prevention and treatment of MS must be prioritized (12). An epidemiological survey conducted in the USA (13) revealed that the risk of cardiovascular and total mortality associated with high SBP in patients with impaired fasting glycemia was significantly increased with the prevalence of MS. The study also revealed that the occurrence of myocardial infarction and stroke can be predicted by the presence of MS in the elderly population. A similar conclusion was also reached in a prospective study in Japan concerning the association between MS and CVD (14). In addition, it was revealed that MS may lead to loss of cognitive function in the elderly.

The number of diabetic patients worldwide has exhibited a more significant growth than that occurring previously, according to a recent study (15). The prevalence of diabetes has also increased significantly. The epidemiologic survey of diabetes in the 14 countries or provinces organized by the CDS during 2007 and 2008 showed that the prevalence of DM in China was $9.7 \%$ in adults $>20$ years old. The total prevalence was $\sim 92.4$ million, which was ranked the first in the world. With the increase in the prevalence of DM, in addition to the increases in efforts to control DM, increased awareness of DM and the prolonged survival time of patients with DM, the prevalence of chronic complications associated with DM has also significantly increased. Epidemiological data revealed that the DR prevalence in patients with type 2 diabetes (T2DM) was $25 \%$ on a global scale. Chronic complications of diabetes may be associated with a number of other factors, but there is a lack of well-designed, large-scale epidemiological data in China at present. The available information is limited, exists within a small range and is significantly different among regions. A study by Li et al (16) showed that the prevalence of DR was $51.3 \%$ in Chinese diabetic patients, and the prevalence increased alongside increases in the number of patients with DM and prolonged survival time. A clinical study by Riqiu Chen et al (17) showed that the prevalence of DN and DR was 36.47 and $28.86 \%$, respectively. In the present study, the prevalence of DN was $47.5 \%$. The prevalence of DN among the male subjects $(53.33 \%)$ was significantly higher than that among the females (40.00\%). The prevalence of DR was $22.92 \%$ overall, $22.22 \%$ for males and $23.81 \%$ for females. The majority of the factors comprising MS are risk factors for CVDs. A previous study (18) also identified MS to be an independent risk factor for CVDs. It has been indicated that MS may be closely associated with diabetic microangiopathy, and in the study by Isomaa et al (19), the results of the prevalence comparisons of MAU and proteinuria in patients with T2DM showed that the prevalence of T2DM with MS was higher than that of T2DM without MS. In the present study, the CDS diagnostic criteria for MS were used to analyze the clinical data of 240 diabetic patients in Jinzhou. The prevalence of DN was $55.33 \%$ in the MS group and $34.44 \%$ in the non-MS group, indicating a significant difference. The prevalence of DR in the MS group was $26.00 \%$, which was higher than the prevalence in the non-MS group (17.78\%); however, no significant difference was indicated. The prevalence comparisons of DN and DR between different genders in the MS and non-MS groups showed similar results.

A number of studies, including the United Kingdom Prospective Diabetes Study and the Diabetes Control and 
Complications Trial, have shown that the prevalence of microvascular complications associated with diabetes increases significantly with increases in HbAlc and BP (20). The clinical study of Riqiu Chen et al (17) also showed that DN was associated with a number of factors, including age, gender, SBP, TG and TC. DR was positively correlated with age, disease duration and other factors. The results in the present study showed that DN was positively correlated with age, SBP, DBP and serum uric acid, while DR was positively correlated with the duration of diabetes. Epidemiological data, qualitative experiments and clinical studies have confirmed that the pathogenic basis underlying MS is IR and obesity, and it has been suggested that IR is more important than obesity in the pathogenesis of MS (21-23). Lipid metabolism may also be involved in the development of DN. Obesity can cause decreases in afferent arteriole resistance by changing the reabsorption of salt by renal tubules, thereby increasing the GFR; this can lead to DN caused by kidney damage in the long-term future (24). The results in the present study showed that DN was positively correlated with TG and LDL-C and negatively correlated with HDL-C. Thus, reducing MAU by reducing weight and regulating blood fat metabolism may delay the development of DN. The present study also revealed that DR was positively correlated with LDL-C. Diabetic microangiopathy shows certain organ-specific features, which mainly occur in the kidney and retina, but the pathology of the other organs, including the brain and lungs, is not evident. Therefore, further study of the organ-specific mechanisms is a novel perspective for finding targets to prevent and treat diabetic microangiopathy.

Domestic and international data have shown that MS is an important and independent risk factor for DN (25-28). It has been shown that DR is positively correlated with TG and LDL-C and negatively correlated with HDL-C. A previous study also showed that DR had an association with numerous factors, including high BP, lipid disorders and high blood sugar (29). Whether MS was an independent factor required further study. Isomaa et al (19) found that MS was an independent risk factor for DN, while DR had no significant correlation with MS. In the present study, the associations between MS and DN/DR were consistent with the results of the study by Isomaa et al (19).

At present, treatment for MS is mainly initiated to avoid the adverse consequences of the disease, i.e. to prevent the development of clinical CVD and T2DM. Therefore, the treatment of MS was actively based on DM, CVD and the diseases associated with MS (30-33). The basic principles underlying the treatment of MS have reached a consensus, with emphasis on lifestyle intervention, followed by drug treatment. Based on the disease pathogenesis, the prevention and treatment of diabetic microangiopathy have been investigated, and the use of certain pathway inhibitors and Traditional Chinese Medicines has been explored (34). Current strategies include the use of aldose reductase inhibitors, such as tolrestat, and the development of protein kinase C-B subtype-specific inhibitors, such as ruboxistaurin, and inhibitors of diabetic microangiopathy glycation end products (35) and fructose 6-phosphate acyltransferase (a key enzyme of the hexosamine pathway). In recent years, with the increased prevalence of microvascular diseases associated with diabetes, the results of the prevention and treatment of these diseases by Chinese medicines, including puerarin, Ginkgo biloba and curcumin, have been noteworthy. Studies investigating these medicines suggested that they led to a certain degree of improvement in the condition of patients with diabetic microangiopathy.

The results of the present study demonstrated that the prevalence of MS among diabetic patients was high. The prevalence of microvascular complications in the MS group was significantly higher compared to that in the non-MS group. In order to prevent the occurrence and development of diabetic microvascular complications, the active and effective control of the prevalence of MS is necessary.

\section{Acknowledgements}

This study was supported by the Natural Science Foundation of Liaoning Province, China (no. 201202148).

\section{References}

1. Pi-Sunyer X: The metabolic syndrome: how to approach differing definitions. Med Clin North Am 91: 1025-1040, 2007.

2. Gluckman PD, Hanson MA, Bateson P, et al: Towards a new developmental synthesis: adaptive developmental plasticity and human disease. Lancet 373: 1654-1657, 2009.

3. Cavali Mde L, Escrivão MA, Brasileiro RS and Taddei JA. Metabolic syndrome: comparison of diagnosis criteria. J Pediatr (Rio J) 86: 325-330, 2010 (In Portuguese).

4. World Health Organization. Definition, diagnosis and classification of diabetes mellitus and its complications Report of a WHO consultation, Part 1: Diagnosis and classification of diabetes mellitus[M].WHO:Geneva,1999.

5. MS CDS ref-waiting for it in English

6. Mogensen CE, Christensen CK, Vittinghus E: The stages in diabetic renal disease. With emphasis on the stage of incipient diabetic nephropathy. [J].Diabetes,1983,32(suppl 2):64-78.

7. Ford ES, Giles WH and Dietz WH: Prevalence of the metabolic syndrome among US adults: findings from the third National Health and Nutrition Examination Survey. JAMA 287: 356-359, 2002.

8. Hajat C and Shather Z: Prevalence of metabolic syndrome and prediction of diabetes using IDF versus ATPIII criteria in a Middle East population. Diabetes Res Clin Pract 98: 481-486, 2012.

9. Riediger ND and Clara I: Prevalence of metabolic syndrome in the Canadian adult population. CMAJ 183: E1127-E1134, 2011.

10. Jia WP, XiangKS, Chen L, et al: A comparison of the application of two working definitions of metabolic syndrome in Chinese population. Zhonghua Yi Xue Za Zhi 84: 534-538, 2004.

11. Li R, Lv M, Xiao F, et al: Investigation on the prevalence rate of metabolic syndrome among elderly in 5 Chinese urban communities. Xian Dai Yu Fang Yi Xue: 7: 460-462, 2012,

12. Cavali Mde L, Escrivão MA, Brasileiro RS and Taddei JA: Metabolic syndrome: comparison of diagnosis criteria. J Pediatr (Rio J) 86: 325-330, 2010.

13. Martins D, Ani C, Pan D, Ogunyemi O and Norris K: Renal dysfunction, metabolic syndrome and cardiovascular disease mortality. J Nutr Metab 2010: 167162, 2010.

14. Saito I, Iso H, Kokubo Y, Inoue M and Tsugane S: Metabolic syndrome and all-cause and cardiovascular disease mortality: Japan Public Health Center-based Prospective (JPHC) Study. Circ J 73: 878-884, 2009.

15. Ginter E and Simko V: Global prevalence and future of diabetes mellitus. Adv Exp Med Biol 771: 35-41, 2012.

16. Li S, Rao X and Tan X. The pathogenesis of diabetic retinopathy and clinical research progress. Bai Qiu En Yi Xue Yuan Xue Bao 9: 118-120, 2011.

17. Chen R and Guan C. Prevalence and risk factors analysis of chronic complications in tie in patients with Type 2 diabetes mellitus. Xin Nao Xue Guan Bing Fang Zhi 11: 429, 2011.

18. McNeill AM, Rosamond WD, Girman CJ, et al: The metabolic syndrome and 11-year risk of incident cardiovascular disease in the atherosclerosis risk in communities study. Diabetes Care 28: 385-390, 2005. 
19. Isomaa B, Henricsson M, Almgren P, et al: The metabolic syndrome influences the risk of chronic complications in patients with type II diabetes. Diabetologia 44: 1148-1154, 2001

20. No authors listed. Intensive blood-glucose control with sulphonylureas or insulin compared with conventional treatment and risk of complications in patients with type 2 diabetes. UK Prospective Diabetes Study (UKPDS) group. Lancet 352: 837-853, 1998.

21. Lee IT, Chiu YF, Hwu CM, et al: Central obesity is important but not essential component of the metabolic syndrome for predicting diabetes mellitus in a hypertensive family-based cohort. Results from the Stanford Asia-pacific program for hypertension and insulin resistance (SAPPHIRe) Taiwan follow-up study. Cardivasc Diabetol 11: 43, 2012.

22. Gallagher EJ, Leroith D and Karnieli E: The metabolic syndrome - from insulin resistance to obesity and diabetes. Med Clin North Am 95: 855-873, 2011.

23. Barth RJ: Insulin resistance, obesity and the metabolic syndrome. S D Med Spec No: 22-27, 2011.

24. No authors listed. The effect of intensive treatment of diabetes on the development and progression of long-term complications in insulin-dependent diabetes mellitus. The Diabetes Control and Complications Trial Research group. N Engl J Med 329: 977-986, 1993.

25. Duran-Perez EG, Almeda-Valdes P, Cuevas-Ramos D, Campos-Barrera E, Muñoz-Hernandez L and Gomez-Perez FJ: Treatment of metabolic syndrome slows progression of diabetic nephropathy. Metab Syndr Relat Disord 9: 483-489, 2011.

26. Maric C and Hall JE: Obesity, metabolic syndrome and diabetic nephropathy. Contrib Nephrol 170: 28-35, 2011.

27. Thorn LM, Forsblom C, Wadén J, Saraheimo M, Tolonen N, Hietala K and Groop PH; Finnish Diabetic Nephropathy (FinnDiane) Study Group: Metabolic syndrome as a risk factor for cardiovascular disease, mortality and progression of diabetic nephropathy in type 1 diabetes. Diabetes Care 32: 950-952, 2009.
28. Martínez Castelao A: Advances in diabetes mellitus, diabetic nephropathy, metabolic syndrome and cardio-vascular-renal risk. Nefrologia 28 (Suppl 5): 79-84, 2008 (In Spanish).

29. Raman R, Gupta A, Pal SS, Ganesan S, Venkatesh K, Kulothungan V and Sharma T: Prevalence of Metabolic Syndrome and its influence on microvascular complications in the Indian population with Type 2 Diabetes Mellitus. Sankara Nethralaya Diabetic Retinopathy Epidemiology And Molecular Genetic Study (SN-DREAMS, report 14). Diabetol Metab Syndr 2: 67, 2010.

30. Sass DA, Chang P and Chopra KB: Nonalcoholic fatty liver disease: a clinical review. Dig Dis Sci 50: 171-180, 2005.

31. Kolovou GD, Anagnostopoulou KK and Cokkinos DV: Pathophysiology of dyslipidaemia in the metabolic syndrome. Postgrad Med J 81: 358-366, 2005.

32. Orchard TJ, Temprosa M, Goldberg R, Haffner S, Ratner R, Marcovina S and Fowler S; Diabetes Prevention Program Research Group: The effect of metformin and intensive lifestyle intervention on the metabolic syndrome: the Diabetes Prevention Program randomized trial. Ann Intern Med 142: 611-619, 2005.

33. Muzio F, Mondazzi L, Sommariva D and Branchi A: Long-term effects of low-calorie diet on the metabolic syndrome in obese nondiabetic patients. Diabetes Care 28: 1485-1486, 2005.

34. Tuttle KR, Bakris GL, Toto RD, McGill JB, Hu K and Anderson PW: The effect of ruboxistaurin on nephropathy in type 2 diabetes. Diabetes Care 28: 2686-2690, 2005.

35. Hanford LE, Enghild JJ, Valnickova Z, et al: Purification and characterization of mouse soluble receptor for advanced glycation end products (sRAGE). J Biol Chem 279: 50019-50024, 2004. 